\title{
A study on Congestion Control of Wireless Communication Network applying ML and FL Techniques
}

\author{
M. Sudhakar, ${ }^{1}$ Vandana Khare ${ }^{2}$ \\ Department of ECE, CMR College of Engineering \& Technology ${ }^{1}$ \\ Department of ECE, CMR College of Engineering \& Technology, India ${ }^{2}$
}

\begin{abstract}
In Recent years wireless network is the most popular way of communication. For example, internet services in companies, cafes, e-markets and in homes. It must be protected against the malicious users who try to harm the isolation, authenticity and privacy of it. Also there is need of traffic control of information sent over this wireless network. In this research article, a technique for controlling the congestion over the wireless networks is shown and to implement it, machine learning tools and fuzzy logic concepts are applied. Congestion control decision mechanism are Transmission rate, queue size, transmission energy, cost assigned, distance from receiver parameters are necessary to be considered. On calculation of these parameters using fuzzy logic, a desired output for congestion control can be determined and its efficiency is calculated using machine learning tool.
\end{abstract}

Keywords: Nä̈ve Bayes, fuzzy logic, machine learning tools, wireless networks, congestion control.

\section{Introduction}

Wireless Overlay Networks - a hierarchical data structure of room-size and wide area data networks, solve the issue of providing network property to an enormous variety of mobile users in an proficient and ascendable manner [1]. With the growth of wireless communication technology, numerous wireless networks are originated. Numerous networks are dominant within the succeeding generation wireless networks [2]. Typical samples of wireless networks are native WLAN and 3G(Wireless Local Area Network). A vital issue is to assimilate these heterogeneous networks and accomplish the mobile nodes whereas moving across heterogeneous networks with continuity, low latency between networks supported numerous technologies and minimum packet loss [3] [4]. A typical example of wireless network is shown in figure 1.

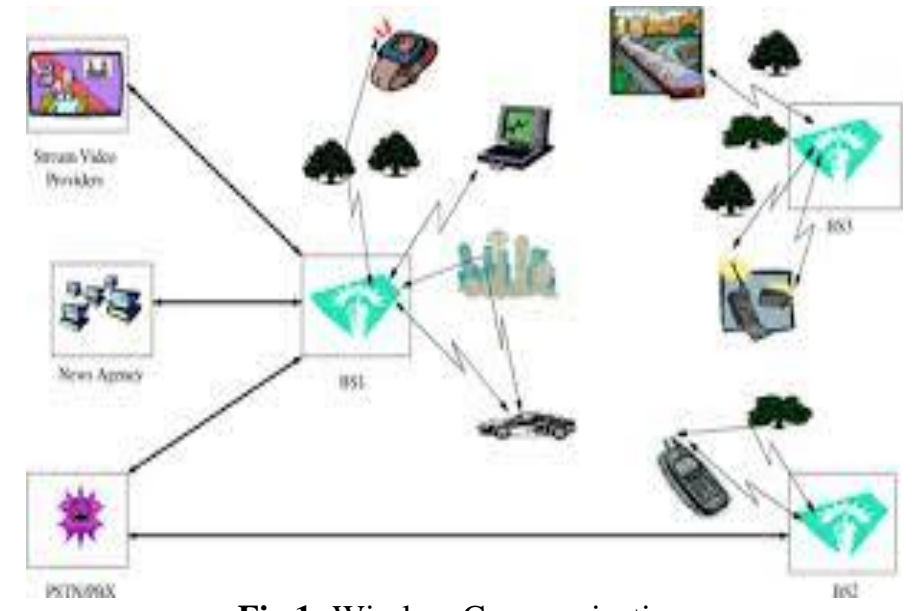

Fig 1: Wireless Communication

Congestion management within the wireless networks is that the foremost downside during this world. In recent Telecommunication, laptop Networks and each wired and wireless communications as well as the net are being supposed for quick transmission of huge quantity of information, that Congestion management is incredibly vital. While not correct Congestion management mechanism, the congestion downfall of such networks would become extraordinarily complicated and could be a real possibility [5]. Congestion management for streamed media traffic over network could be a challenge attributable to the sensitivity of such traffic. This challenge has galvanized the researchers over the last decade to grow variety of congestion management protocols and mechanisms that ensemble the traffic and provides honest repairs for each unicast and multicast communications [4] [6]. 


\section{Materials And Methods}

Fuzzy Logic: It could be a style of many-valued logic that has been employed in networks and has supported to developments in network potency. American state compacts with reasoning that's inaccurate instead of mounted and precise. Fuzzy logic has introduced several strategies for in situ of and deducing from unsure or incomplete data. Fuzzy ideas cannot be incontestable by an easy set inclusion operator $\epsilon$, however there's a degree of membership. In different words, whereas Boolean algebra has simply 2 values, true (always bestowed numerically as 1) or affirmative, and false (always bestowed numerically as 0 ), or No, symbolic logic extends these 2 values to values between zero and one victimization the idea of degrees of membership [7]. Fuzzy Logic System is depicted in the Figure 2 given below.

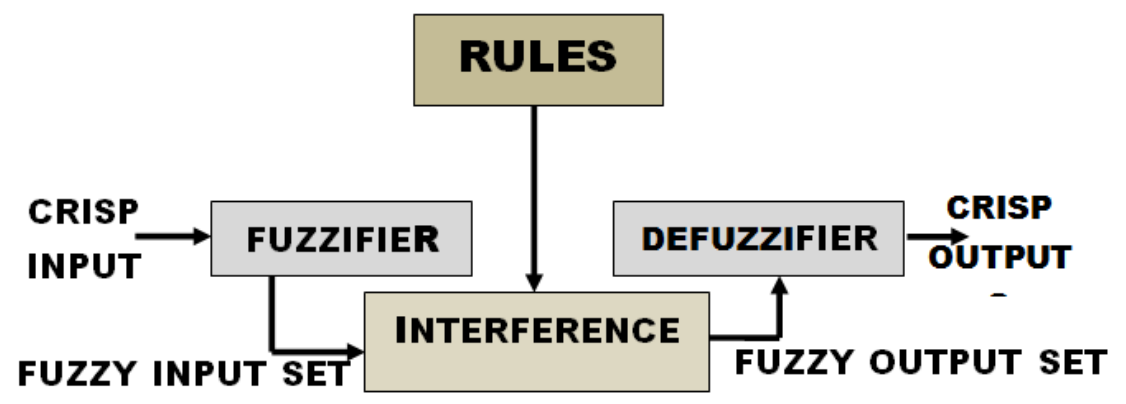

Fig 2: Fuzzy Logic System

Weka Tool: It has been used for coaching and testing of Machine Learning algorithms [9]. This tool could be a gathering of huge variety of Machine Learning algorithms, out of which 5 largely used Machine Learning algorithms are used during this analysis work and performance of these Machine Learning algorithms has been compared on the idea of various parameters. It could be a written in Java, developed at the University of Waikato, Newzealand. It is shown in the figure below. It is free software available in GNU General Public License.

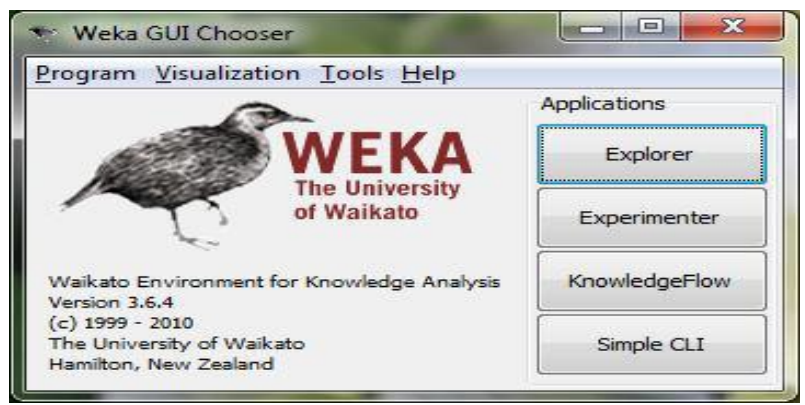

Fig 3: Weka Tool

\section{Proposed System}

In this paper, a manual dataset of 2084 values is formed victimization symbolic logic. The Mat lab utilized in fuzzy is shown in figure 4 below.

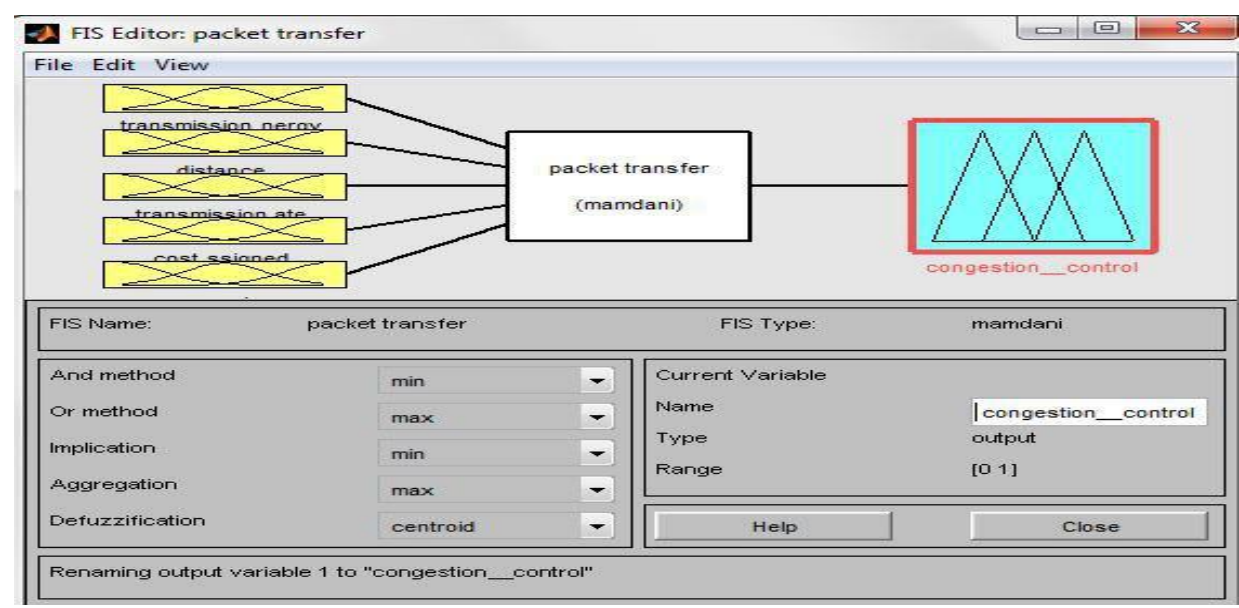

Fig 4: Mat lab utilized in fuzzy 
Five input parameters are taken as Transmission rate, queue size, transmission energy, cost assigned, distance from receiver and output is congestion management. Here, Fuzzy logic has been used, a rule set of 400 values is formed that is shown below.

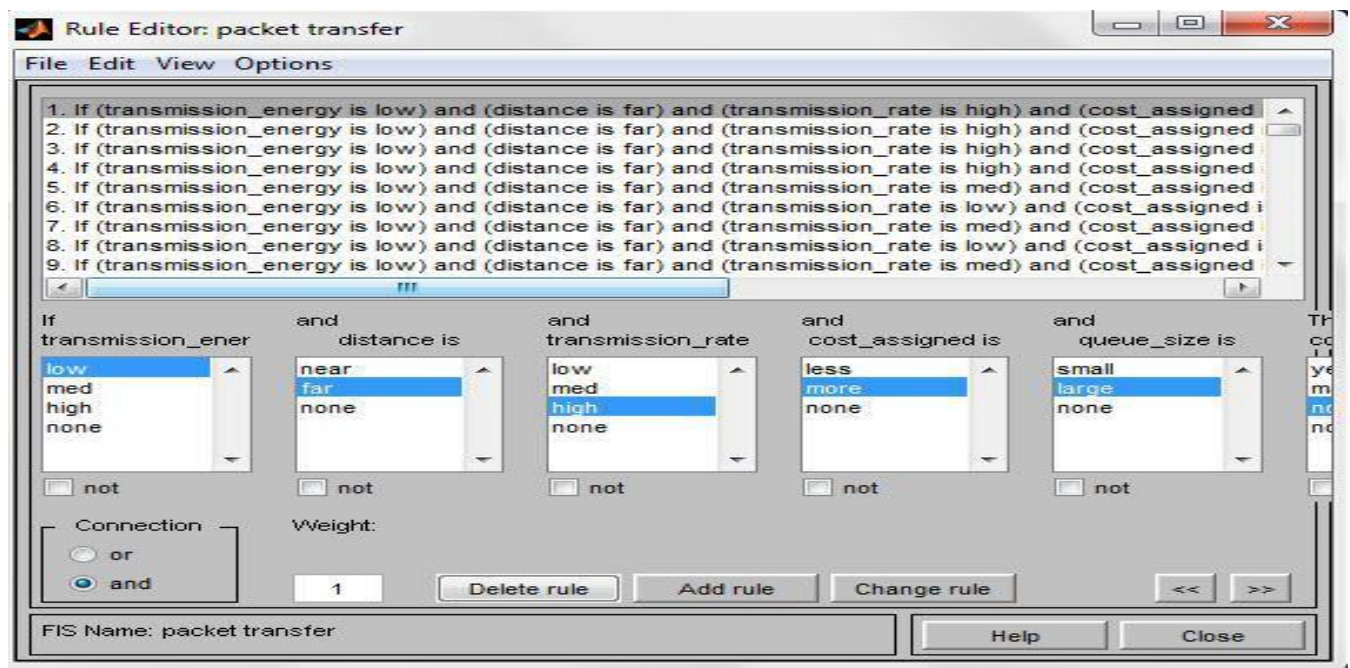

Fig 5: Rule Editor

\section{Implementation And Experiments}

The rule viewer shows the assorted rules consistent with parameters and their impact on the output.

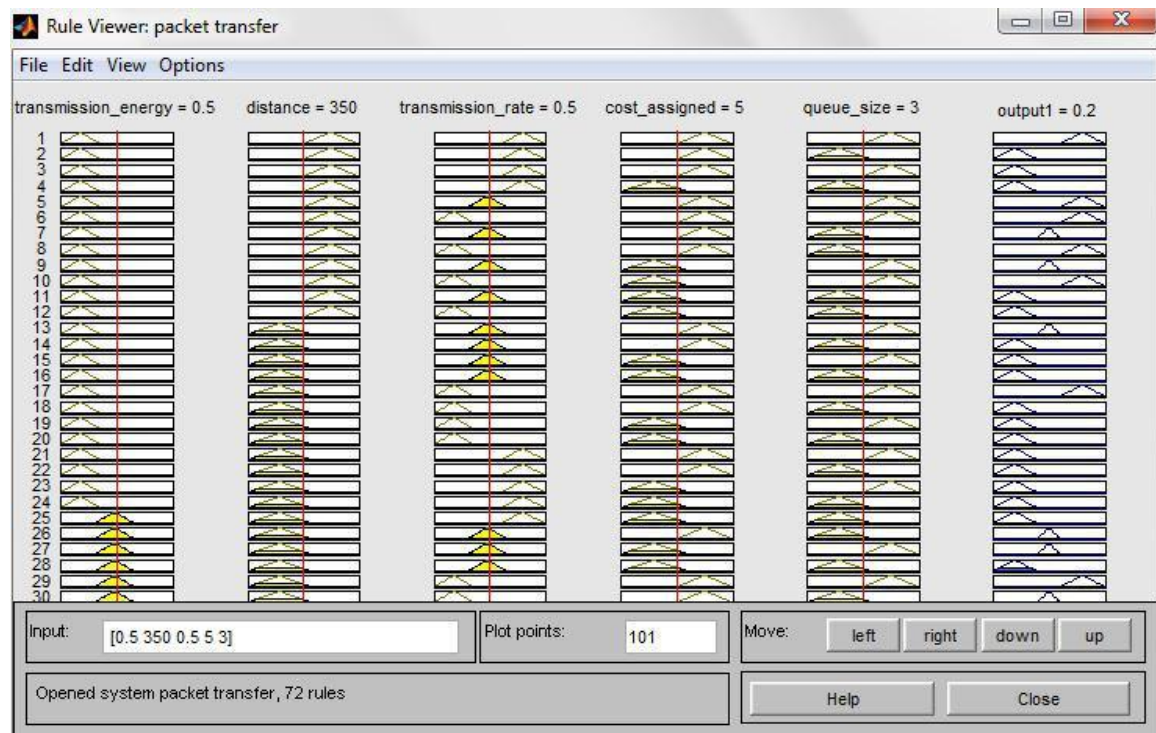

Fig 6: Rule Viewer

Surface Viewer shows the autocorrelation between the parameters chosen and is shown below.
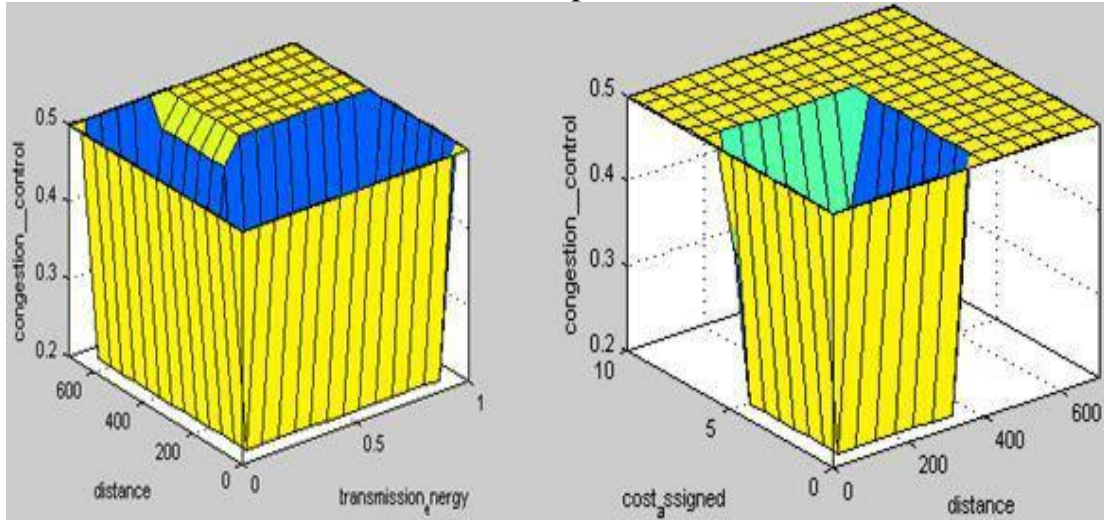

Fig7: Surface Viewer 
Some values of those rules are checked for his or her validation victimization Matlab commands (readfis and evalfis) as shown in table1:

Table 1: Validation Table

\begin{tabular}{|c|c|c|c|c|c|c|}
\hline $\begin{array}{c}\text { Transmission } \\
\text { Energy }\end{array}$ & Distance & Transmission Rate & Cost Assigned & Queue Size & $\begin{array}{c}\text { Congestion } \\
\text { Control }\end{array}$ & Output \\
\hline 0.01 & 510 & 0.1 & 6 & 1 & 0.75 & No \\
\hline 0.02 & 520 & 0.11 & 7 & 5 & 0.75 & No \\
\hline 0.03 & 530 & 0.12 & 1 & 2 & 0.75 & No \\
\hline 0.04 & 540 & 0.13 & 2 & 5.5 & 0.75 & No \\
\hline 0.05 & 550 & 0.4 & 7 & 1 & 0.5 & May be \\
\hline 0.06 & 560 & 0.41 & 8 & 5 & 0.75 & No \\
\hline 0.07 & 570 & 0.42 & 2 & 2 & 0.25 & Yes \\
\hline 0.08 & 580 & 0.43 & 3 & 5.5 & 0.5 & May be \\
\hline 0.09 & 590 & 0.7 & 6 & 1 & 0.25 & Yes \\
\hline 0.1 & 600 & 0.71 & 7 & 5 & 0.5 & May be \\
\hline
\end{tabular}

Transmission rate, queue size, transmission energy, cost assigned, distance from receiver.

The membership functions of varied input parameters are given as follows:

Data Transmission Rate: While broadcast and accept stations have been interconnected by a call, a training sequence for the modem is executed and, also, a test signal transmission sequence is affected to set up a data transmission rate before delivery of information. Data transmission rate should be fast so that delivery of packets at the destination will be received on time. Quick and fast data transmission rate are assigned as lower link cost in wireless networks. Training on the transmission rates which a modem can use is executed satisfying a predetermined protocol and without increasing the protocol time.

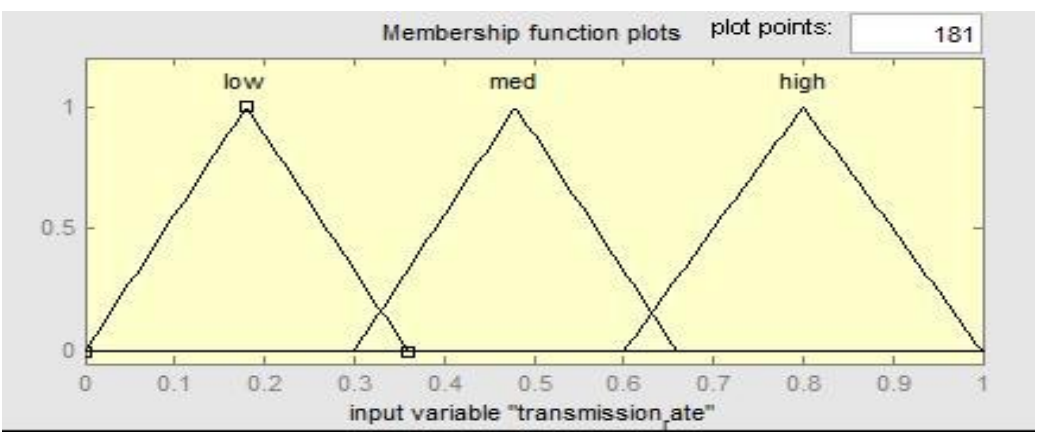

Fig 8: Membership function for Data Transmission Rate

Queue size: The input fuzzy variable "queue size" indicates the buffer capacity at any node. This parameter helps avoid packet drops due to congestion at the receiver. Congestion leads to great trouble for efficient transmission of data packets at receiver end in networks. This queue size of packets should be small so that there will be less waiting time for execution.

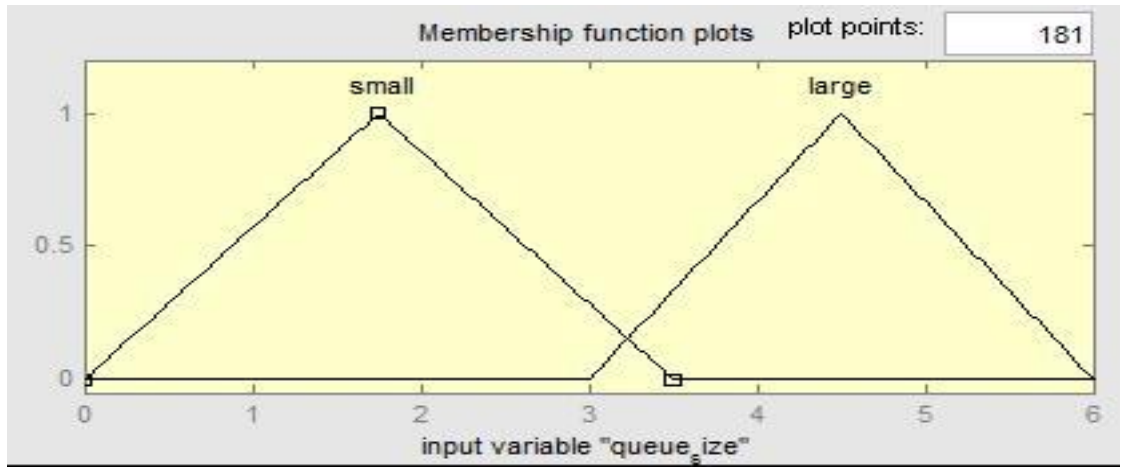

Fig 9: Membership function for queue size

Transmission energy: It is instantly accustomed transmit packets over a wireless link in WSN. The main key observation is that the energy required to transmit a packet can be significantly reduced and this parameter is lowering the transmission power and transmitting the packet over a longer period of time in wireless networks. It represents the energy needed to transmit a data packet from node one to another node. Lower value of transmission energy leads to lower congestion. 


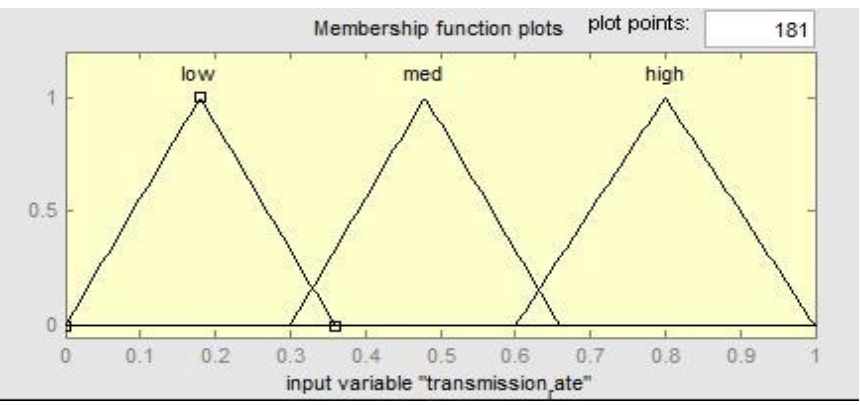

Figure 10: Membership function for Transmission energy

Cost assigned to each path: Each sensor node is assigned a dynamic weight depending upon its current status. An in-active node that is neither sensing nor relaying is assigned a highest value whereas a node that is performing both these tasks is assigned a least weight. This parameter helps in selecting nodes which are either inactive or are only in the sensing state. A high value of weight makes the node favourable for next-hop, resulting in a lower value of link cost in wireless networks.

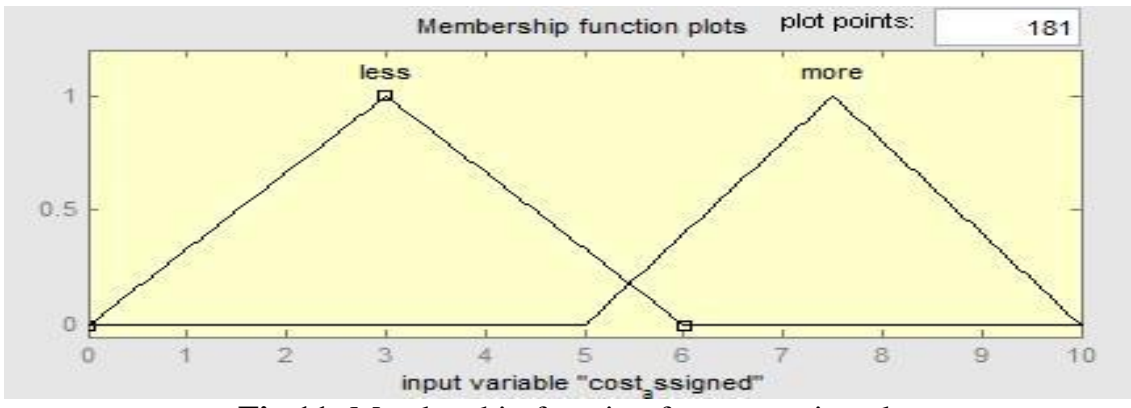

Fig 11: Membership function for cost assigned

Distance between transmitter and receiver: The fuzzy input variable "Distance between transmitter and receiver," enables selection of routes with minimum hops. Nodes nearer to the gateway are thus assigned lower link cost. Minimum number of hops provides an efficient way for transmission of packets at destination and it also reduces the complexity of sensor networks.

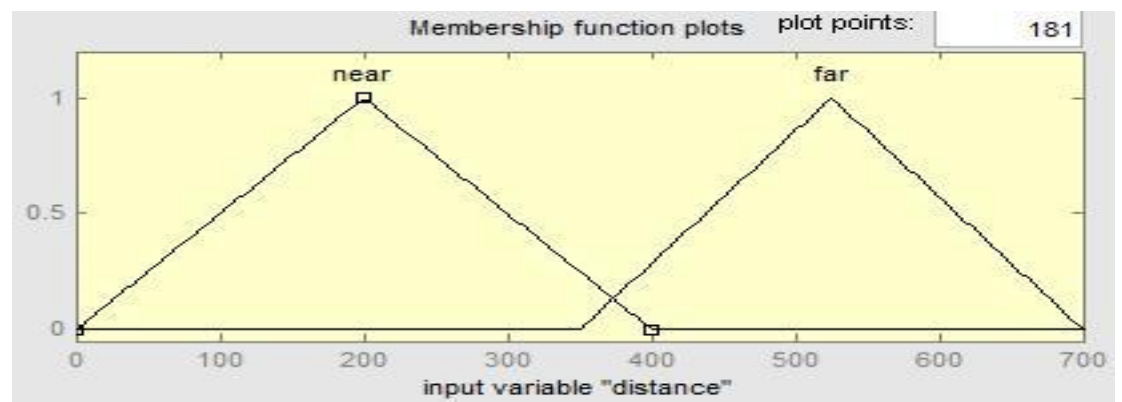

Fig 12: Membership function for distance

Output: Output here is congestion control and the membership function taken here are: 'Yes', 'May be' and 'No'.

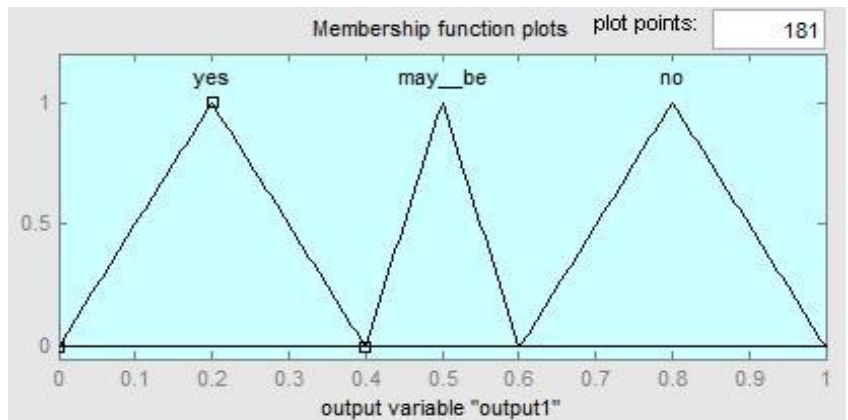

Fig 13: Membership function for output 


\section{Results}

Five machine learning tools are used i.e. Bayesnet, Naïve Bayes, C4.5 call tree, Back propagation, Radial Basis perform. Weka Tool is employed for locating the classification accuracy and coaching time of those tools to seek out the most effective among them. Among 2084 rules, 1784 are used for coaching knowledge and three hundred are used for testing the information.

Table 2: Table showing the performance of the 5 neural tools

\begin{tabular}{|l|c|c|c|c|c|}
\hline & Bayes net & $\begin{array}{c}\text { Naive } \\
\text { Bayes }\end{array}$ & C4.5 Decision Tree & $\begin{array}{c}\text { Back Propagation } \\
\text { Function }\end{array}$ & $\begin{array}{c}\text { Radial Basis } \\
\text { Training time(sec) }\end{array}$ \\
\hline Classification Accuracy 9\%) & 9.13 & 0.02 & 0.06 & 3.42 & 3.0 \\
\hline
\end{tabular}

training time(secs)

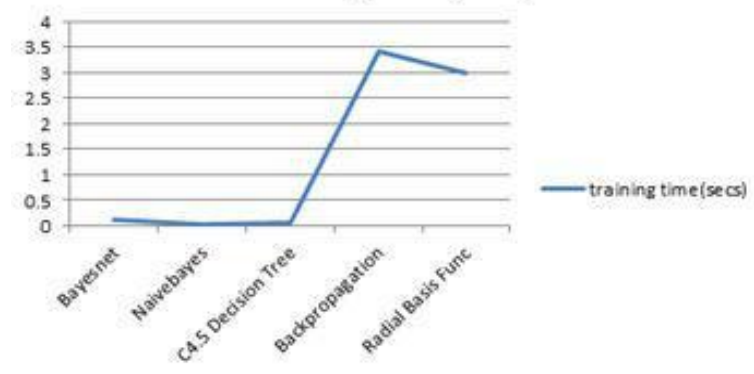

\section{classificationaccuracy}

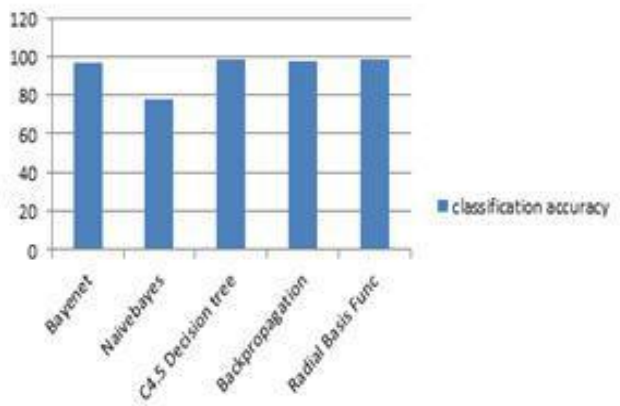

Fig 14: Graphs showing the comparison between the 5 neural tools

Using the coaching and testing knowledge, graphs showing the comparison between the coaching time and classification accuracy of the tools are drawn.

\section{Conclusion}

Congestion management is that the major downside in wireless knowledge transfer. To stay a check on this downside, a brand new technique has been presented in this paper that uses symbolic logic and machine learning tools. On the comparison of those neural tools, it may be ascertained that C4.5 is showing better results among all of the 5 tools i.e. the classification accuracy given by $\mathrm{C} 4.599 \%$ that is most among all whereas victimization the coaching time of $\mathrm{C} 4.5$ is 0.06 seconds.

\section{References}

[1]. Xuekang, S., G. Wanyi, X. Xingquan, X. Baocheng, \& G. Zhigang (2009). Node discovery algorithm based multipath olsr routing protocol. Information Engineering, International Conference on 2, 139-142.

[2]. Manoj Sharma, Dr. R.K.Khola, "Fuzzy logic based handoverdecision system", International Journal of Ad hoc, Sensor \& Ubiquitous Computing (IJASUC) Vol.3, No.4, August 2012.

[3]. Ali Murtaza, Mansoor Ahmed, "Decision algorithm and procedure for fast handover between 3G and WLAN",Master's Thesis in Computer Network Engineering, School of Information Science, Computer and Electrical Engineering, Halmstad University

[4]. M. Sudhakar, (2015). Wmns: Efficient Scheduling Algorithm and Classification of Routers With Multiple Gateways. International Journal of Applied Engineering Research ISSN 0973-4562 Volume 10, Number 7 (2015) pp. 17213-17228 @ Research India Publications.

[5]. Yi, J., A. Adnane, S. David, \& B. Parrein (2010). Multipath Optimized Link State Routing for Mobile ad hoc Networks. Ad Hoc Networks.

[6]. M. Sudhakar, (Mar-Apr 2016), A Study Of Wireless Mesh Networks Insider Attacks Of Selective Jamming Or Dropping, IOSR Journal of Electronics and Communication Engineering, e-ISSN: 2278-2834, p- ISSN: 2278-8735.Volume 11, Issue 2, Ver. I (MarApr .2016), PP 60-66.

[7]. Maimour, M. (2008). Maximally radio-disjoint multipath routing for wireless multimedia sensor networks. In WMuNeP '08: Proceedings of the 4th ACM workshop on Wireless multi-media networking and performance modeling, New York, NY, USA, pp. 26-31. ACM.

[8]. Cagatay Sonmez, Ozlem Durmaz Incel, Sinan Isik, Mehmet Yunus Donmez and Cem Ersoy, "Fuzzy-based congestion control for wireless multimedia sensor networks", Sonmez et al. EURASIP Journal on Wireless Communications and Networking 2014.

[9]. L.A. Zadeh, "Fuzzy Algorithms", Information and Control, vol. 12, issue. 2, pp. 94-102,1968

[10]. http://www.cs.waikato.ac.nz/ml/weka/ 


\section{AUTHORS BIOGRAPHY}

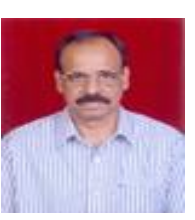

Dr. M. Sudhakar': Graduated from JNTU College of Engineering, Hyderabad in 1979, with specialization in ECE. He completed his M.Tech from Indian Institute of Technology Madras in 1986 with the specialization in Instrumentation, Control \& Guidance. Obtained doctoral degree from Annamalai University. Successfully headed R\&D Project assigned by IAF on "Mathematical Modelling \& Simulation of Aero Engine Control System" at Aeronautical Development Establishment, Bangalore and Gas Turbine Research Establishment, Bangalore. He is presently working as a Professor in the department of ECE and Dean (Planning \& Development) at CMR College of Engineering \& Technology, Hyderabad. He is a Fellow member of IETE and Life member of ISTE.

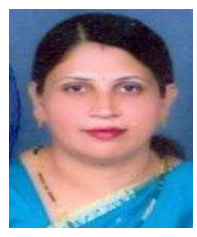

Mrs. Vandana Khare ${ }^{2}$ submitted her Ph D thesis in Communication Engineering at JNTU Hyderabad. She completed M.E (Digital techniques) in 1999 from SGSITS, Indore (M.P) India and B.E in ECE in the year 1994 from GEC Rewa (M.P). She is Associate Professor in ECE at CMR College of Engineering \& Technology. Secunderabad. She has 18 years of teaching experience and has published 14 research papers in International journals \& presented 5 papers in National \& International conferences. She is life member of ISTE, IETE \& IEEE Technical societies. Her research Interest includes Wireless Communication, Computer Networks, Mobile Computing and Bio-Medical Imaging. 